\title{
Laser frequency stabilization based on a universal sub-Doppler NICE-OHMS instrumentation for the potential application in atmospheric lidar
}

\author{
Yueting Zhou ${ }^{1,5}$, Jianxin Liu ${ }^{1,5}$, Songjie Guo ${ }^{1,5}$, Gang Zhao ${ }^{1,5}$, Weiguang Ma $^{1,5}$, Zhensong Cao ${ }^{2}$, Lei Dong ${ }^{1,5}$, \\ Lei Zhang ${ }^{1,5}$, Wangbao Yin ${ }^{1,5}$, Yongqian $\mathrm{Wu}^{3}$, Lianxuan Xiao ${ }^{1,5}$, Ove Axner ${ }^{4}$, and Suotang Jia ${ }^{1,5}$ \\ ${ }^{1}$ State Key Laboratory of Quantum Optics \& Quantum Optics Devices, Institute of Laser Spectroscopy, \\ Shanxi University, Taiyuan, 030006, China \\ ${ }^{2}$ Key Laboratory of Atmospheric Composition and Radiation, Anhui Institute of Optics and Fine Mechanics, \\ Chinese Academy of Sciences, Hefei, 230031, China \\ ${ }^{3}$ Institute of Optics and Electronics, Chinese Academy of Sciences, 610209, Chengdu, China \\ ${ }^{4}$ Department of Physics, Umeå University, 90187 Umeå, Sweden \\ ${ }^{5}$ Collaborative Innovation Center of Extreme Optics, Shanxi University, Taiyuan, 030006, China
}

Correspondence: Weiguang Ma (mwg@sxu.edu.cn)

Received: 30 October 2018 - Discussion started: 30 November 2018

Revised: 22 February 2019 - Accepted: 4 March 2019 - Published: 19 March 2019

\begin{abstract}
Lidar is an effective tool for high-altitude atmospheric measurement in which a weak absorption line for the target gas is selected to ensure a large optical depth. The laser frequency stabilization to the line center is required, and a sub-Doppler ( $\mathrm{sD}$ ) spectroscopy of the target line is preferred as a frequency reference. In this paper, a novel universal $\mathrm{sD}$ noise-immune cavity-enhanced optical heterodyne molecular spectroscopy (NICE-OHMS) instrumentation based on a fiber-coupled optical single-sideband electro-optic modulator (f-SSM) for the potential application in atmospheric lidar for different target gases with different types of lasers is reported. The f-SSM can replace all frequency actuators in the system, so as to eliminate the individual design of feedback servos that often are tailored for each laser. The universality of the instrumentation was demonstrated by the alternative use of either an Er-doped fiber laser or a whispering-gallerymode laser. Then the instruments based on both lasers were used to produce the SD signals of acetylene, which worked as a frequency reference to stabilize the laser. By performing the lockings, relative frequency stabilizations of $8.3 \times 10^{-13}$ and $7.5 \times 10^{-13}$ at an integration time of $240 \mathrm{~s}$ were demonstrated.
\end{abstract}

\section{Introduction}

The atmospheric lidar can study the atmospheric properties from the ground up to the top of the atmosphere by using a laser. In order to obtain a large dynamic range in height, a relatively weak transition should be selected to ensure a large optical depth. Meanwhile, the laser frequency should be stabilized to the line center for a valid detection. In reality, the required frequency stability ranges from hundreds of kilohertz to dozens of megahertz, which relies on the line width of the target gas spectrum (Ehret et al., 2008). Up to now, the error for frequency stabilization in most of the lidar system is measured by a high-precision wavelength meter, which strongly depends on the stability of the wavelength meter. A local absorption spectroscopy of target gas is another alternative reference for active frequency stabilization. Therefore, the Jet Propulsion Laboratory in the US has designed a frequency stabilization system for coherent lidar application with a frequency error of $2.06 \mathrm{MHz}$ for detection of the $v_{1}+v_{3}$ band of acetylene (Meras et al., 2008). A group at the University of Maryland has designed another frequency stabilization system with frequency drift better than $0.3 \mathrm{MHz}$ for the detection of $\mathrm{CO}_{2}$ at the wavelength of $1572 \mathrm{~nm}(\mathrm{Nu}-$ mata et al., 2011). Benefiting from the narrow width of sub- 
Doppler (sD) absorption spectroscopy, it is the most suitable reference to obtain a high-quality frequency stabilization.

$\mathrm{SD}$ spectroscopy is also an important tool for a number of other scientific research fields, including assessments of molecular spectra, investigation of chemical kinetics, realization of frequency standards and assessment of fundamental constants (Moretti et al., 2013; Parthey et al., 2011; Tarallo et al., 2011; Taubman et al., 2004). sD spectroscopy provides responses with high spectral resolution that are free from Doppler broadening ( $\mathrm{Db}$ ). To obtain such signals, high laser powers and low-pressure conditions are normally used. To circumvent the use of such lasers, sD spectroscopy can be performed in Fabry-Pérot (FP) cavities since such cavities can provide intracavity powers that are significantly higher than that of the impinging light field (by a factor of $F / \pi$, where $F$ is the finesse of the cavity) (Delabachelerie et al., 1994; Ishibashi and Sasada, 1999). Since high-finesse FP cavities have narrow transmission modes, for most types of applications, the frequency of the laser field needs to be locked to that of the cavity mode, often by use of the PoundDrever-Hall (PDH) technique (Drewer et al., 1983).

A drawback when absorption spectroscopy (AS) is combined with high-finesse cavities is that, in its simplest realization, i.e., when cavity-enhanced direct absorption spectrometry is performed, it is susceptible to frequency-to-amplitude converted noise caused by the residual frequency jitter between the frequencies of the laser field and the cavity mode that deteriorates the signal (Ye et al., 1996). As a means to circumvent this, the technique of noise-immune cavityenhanced optical heterodyne molecular spectroscopy (NICEOHMS) was invented. In this, the laser light is modulated at a radio frequency (RF) to perform frequency modulation spectroscopy (FMS). By locking the modulation frequency of the FMS to the free spectral range (FSR) of the cavity, NICE-OHMS does not only benefit from the ability of FMS to reduce $1 / f$ noise, which is often the dominating type of noise of the laser intensity, it also provides immunity to the frequency-to-amplitude noise (Axner et al., 2014; Ma et al., 1999, 2016; Ye et al., 1996). It therefore has large potential for a variety of applications.

NICE-OHMS was first realized by Jun Ye et al. in 1996 for frequency reference purposes (Ye et al., 1996). By use of an extremely well-stabilized fixed-frequency Nd:YAG laser and a cavity with a finesse of $10^{5}$, a $\mathrm{C}_{2} \mathrm{HD}$ transition at $1064 \mathrm{~nm}$ was detected by use of a $\mathrm{SD}$ response. This first realization of NICE-OHMS demonstrated an unprecedented detection sensitivity of $1 \times 10^{-14} \mathrm{~cm}^{-1}$ at $1 \mathrm{~s}$ averaging time.

A variety of tunable lasers, including an external cavity diode laser (ECDL) (Bell et al., 2009; Chen and Liu, 2015; Dinesan et al., 2014; Ishibashi and Sasada, 2000; Saraf et al., 2016; van Leeuwen and Wilson, 2004), quantum cascade laser (QCL) (Taubman et al., 2004), optical parametric oscillator (OPO) (Hausmaninger et al., 2015; Talicska et al., 2016; Taubman et al., 2004), quantum-dot laser (Chen and Liu, 2017), distributed feedback laser (DFB) (Foltynow- icz et al., 2010) and whispering-gallery-mode (WGM) laser (Zhao et al., 2017), have been applied to the NICE-OHMS system over the years. The most persistent development of NICE-OHMS has been performed based on erbium-doped fiber lasers (EDFLs), which lase in the near-IR (NIR) region (Ehlers et al., 2012; Schmidt et al., 2007). This has opened up for the use of fiber-coupled optical components, e.g., fibercoupled acoustic-optic modulators (f-AOMs) to expand the bandwidth for PDH locking and fiber-coupled electro-optic modulators (f-EOMs) that can be driven by fairly low RF powers. This has simplified, to a large extent, the experimental setup and improved the applicability of NICE-OHMS.

Despite the fact that the NICE-OHMS technique has been stated to be noise immune, the immunity is adversely affected when a NICE-OHMS signal is detected, either that from the molecular species under scrutiny or that from the optical system. Under these conditions, the quality of the detected signal can be limited by the performance of the locking procedure (Ma et al., 2016; Schmidt et al., 2010). This implies that it is of importance to lock the frequency of the laser to that of the cavity as tightly as possible. This requires both a sufficiently large locking bandwidth and a high gain at low frequencies. In order to provide the former, more than one frequency actuator is usually utilized, often a piezoelectric transducer (PZT) inside the laser and an external AOM. In addition, to prevent oscillations that can take place close to resonances of the actuators, in particular those from PZT, individual designs of proportional integral differential (PID) servos, specifically tailored for each laser, need to be developed and realized. This significantly complicates the construction of the NICE-OHMS system and impairs its applicability (Ehlers, 2014; Foltynowicz, 2009).

In recent years, a commercial fiber-coupled optical singlesideband electro-optic modulator (f-SSM), sometimes in the literature also referred to as an optical single-sideband suppressed-carrier modulator (Loayssa et al., 2001), has been used to shift the laser frequency with considerable tuning range and large modulation bandwidth. Such a device is composed of two Mach-Zehnder interferometers, each driven by a RF. By adjusting the phase difference of the two RF signals it can be made to output a single sideband (Gatti et al., 2015). As a universal frequency shifter, it can replace all other frequency actuators in NICE-OHMS that are used for controlling the frequency of the laser light. Therefore this can facilitate the realization of a universal NICE-OHMS system that can be used with a variety of lasers.

This paper presents a universal NICE-OHMS setup for sD spectroscopy based on an f-SSM (MU-SSB-N-15-PMFCAPC, Beijing Keyang Photonics Co. Ltd., China) for potential application in the atmospheric lidar. The universality of this spectrometer is demonstrated by the alternative use of an EDFL (Adjustik E15 PztS PM, NKT Photonics, Denmark) and a WGM laser (OE4020-153806-PA02, OEwaves, USA). The applicability of the instrumentation was demonstrated by detection of SD NICE-OHMS signals of acety- 
lene from both lasers, at 1531 and $1538 \mathrm{~nm}$. They were then worked as references to stabilize the laser output frequencies to the target acetylene transitions. From 2014 to 2017, NICEOHMS-technique-based frequency stabilizations have been performed by several groups, the relative frequency stabilities of $5 \times 10^{-13}$ (Dinesan et al., 2014, 2015) and $6 \times 10^{-11}$ (Chen and Liu, 2017) have been demonstrated for an integration time of $1 \mathrm{~s}$, which is suitable for future applications in the atmospheric lidar.

\section{Experimental setup and PDH locking performance}

The experimental setup is shown in Fig. 1. The output of the laser passed through, in sequence, an f-SSM driven by a voltage-controlled oscillator (VCO1, SG386, Stanford Research Systems, USA), a fiber-coupled erbium-doped fiber amplifier (f-EDFA) that is used to amplify and stabilize the laser power and a f-EOM with proton-exchanged waveguide (to suppress the residual amplitude modulation, RAM) ( $\mathrm{Si}$ lander et al., 2012) before it entered a fiber-coupled collimator (f-C) to be sent into free space. This implies that the laser frequency could be detuned by changing the frequency of the RF signal provided by the VCO1. Two modulation frequencies, 25.16 and $380.16 \mathrm{MHz}$, were imposed on the laser light by the use of a single f-EOM for the PDH locking and the FMS, providing modulation indices of 0.19 and 0.8 , respectively (Silander et al., 2012). Before impinging onto the cavity, the laser passed through a mode-matching lens (MML), a half-wave plate $(\lambda / 2)$, a polarization beam splitter (PBS) and a quarter-wave plate $(\lambda / 4)$. Both of the Lorenzian line widths of the applied EDFL and WGM lasers are on the order of $100 \mathrm{~Hz}$.

The cavity was composed by a plane and a concave mirror with a finesse of 2300 (estimated by cavity ring-down measurement) and cavity modes with a full width at half maximum (FWHM) of $160 \mathrm{kHz}$. A total of $52 \%$ of the incident optical power was coupled into the cavity. The two mirrors were glued on two PZTs, which, in turn, were glued to the cavity spacer, which was made of Zerodur. The mirror distance was $39.4 \mathrm{~cm}$, which gave rise to a FSR of $380.16 \mathrm{MHz}$. During the measurement, the averaged temperature stability of the cavity is better than $0.5 \mathrm{~K} \mathrm{~h}^{-1}$ since the cavity is exposed in the air.

The reflection of the cavity was deflected by the PBS to a photodetector (PD1) for the PDH and the DeVoe-Brewer (DVB) lockings (DeVoe and Brewer, 1984), the latter used to lock the modulation frequency of the FMS to the FSR of the cavity. The light transmitted through the cavity was focused onto a photodetector (PD2), which provided the NICEOHMS signal. To minimize the amount of background signals from etalons, the optical components were, as much as possible, tilted and placed at an etalon-immune distance (EID) (Ehlers et al., 2014).
To avoid the complex design of a bandpass filter working in the RF frequency region, a $25.16 \mathrm{MHz}$ signal for the PDH locking was created from a low-pass-filtered beat signal between two RF frequencies (at 380.16 and $355 \mathrm{MHz}$ ) that were generated by two RF generators (Bell et al., 2009). The error signal for the PDH locking was obtained by demodulating the reflected light from the cavity at $25.16 \mathrm{MHz}$, which then was sent to the VCO1 through a proportionalintegral-derivative servo (PID1). The design of this servo was thereby only dictated by the frequency response of the VCO1 and not by those of the lasers. To provide high gain in the low-frequency region, the locking servo contained two integrators.

It should be noted that although both aforementioned lasers can be detuned by a PZT inside the laser, the two PZTs have different frequency responses: low-pass frequency corners at 4.5 and $50 \mathrm{kHz}$ and oscillation frequencies at 34 and $48 \mathrm{kHz}$ for the EDFL and at $38 \mathrm{kHz}$ for the WGM laser. Therefore, in conventional setups, careful designs of locking servos (with individually optimized parameter values) and an external f-AOM would have been needed (the latter to expand the locking bandwidth, preferably up to around $100 \mathrm{kHz}$ ). In our system, however, the employment of a f-SSM as a unique and fast-frequency shifter can eliminate the individually designed $\mathrm{PDH}$ servos that are needed for different types of lasers.

Figure 2 shows the frequency noise spectra of the in-loop error signals for PDH locking, which is measured by an electronic spectrum analyzer (FSW, Rohde \& Schwarz, Germany). The black and red lines represent the situations for the EDFL and the WGM lasers, respectively, measured without any change in servo parameters for the PDH locking. Both the video bandwidth (VBW) and resolution bandwidth $(\mathrm{RBW})$ in the measurement are $30 \mathrm{~Hz}$. It can be seen that both spectra have a similar shape and that the servo reduces the noise in the low-frequency region efficiently due to the use of two integrators in series. The peaks at 19 and $38 \mathrm{kHz}$ come from the common ground in the lab, which has been found difficult to eliminate, while the bumps at $200 \mathrm{kHz}$ indicate the bandwidth of the PDH servo (Fox et al., 2002). The difference between the two curves is attributed to dissimilar phase noises of the two lasers. This demonstrates that the locking bandwidth is $200 \mathrm{kHz}$ irrespective of which laser the NICE-OHMS system has utilized.

It is worth noting that the PDH locking bandwidth is mainly limited by the VCO1, which in this work had a bandwidth of $100 \mathrm{kHz}$. As suggested by Gatti et al. (2015) the bandwidth of the PDH servo can be increased to $5 \mathrm{MHz}$ if a higher-bandwidth VCO and a good PID servo were employed. Such an action will expand the applicability of the NICE-OHMS system to lasers with larger line widths, e.g., DFB and ECDL. 


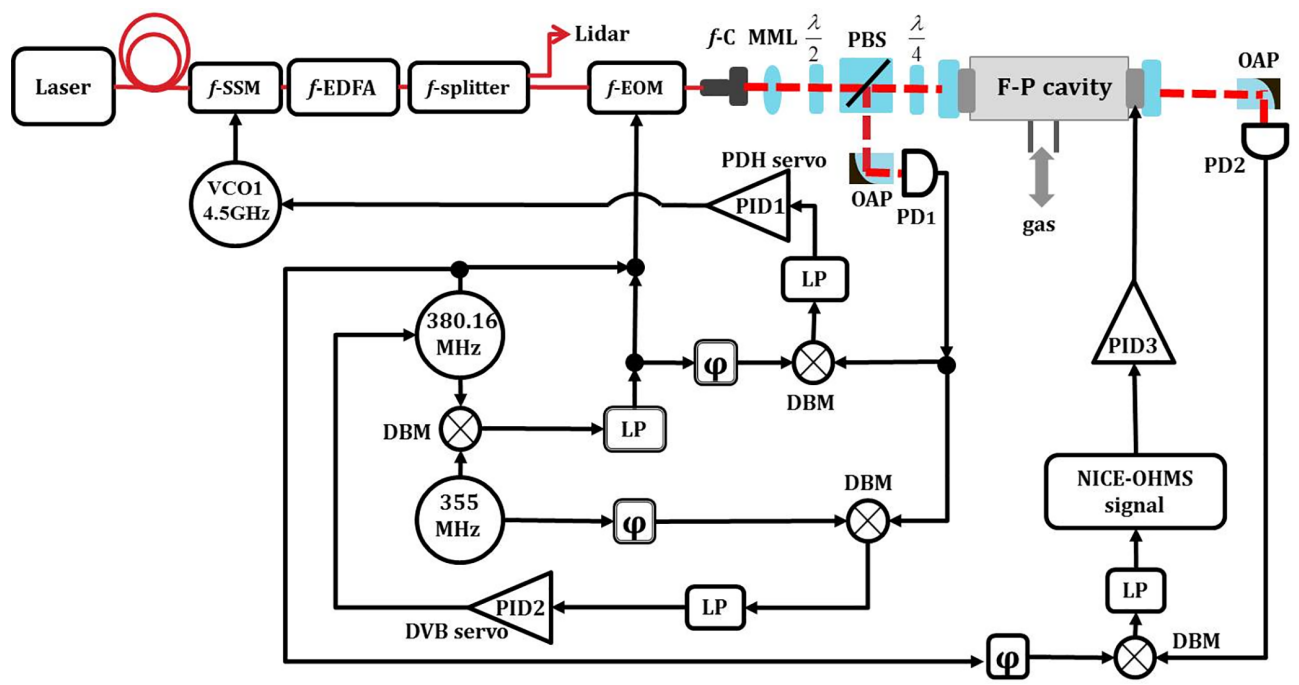

Figure 1. Experimental setup: f-SSM, fiber-coupled single-sideband electronic optical modulator; f-EDFA, fiber-coupled erbium-doped fiber amplifier; f-EOM, fiber-coupled electronic optical phase modulator; f-C, fiber collimator; MML, mode-matching lens; $\lambda / 2$, half-wave plate; PBS, polarizing beam splitter; $\lambda / 4$, quarter-wave plate; OAP, off-axis parabolic mirror; PD, photodetector; LP, low-pass filter; PID, proportional-integral-derivative controller; VCO, voltage control oscillator; DBM, double balanced mixer; $\varphi$, phase shifter; DVB servo, DeVoe-Brewer servo; F-P cavity, Fabry-Pérot cavity.

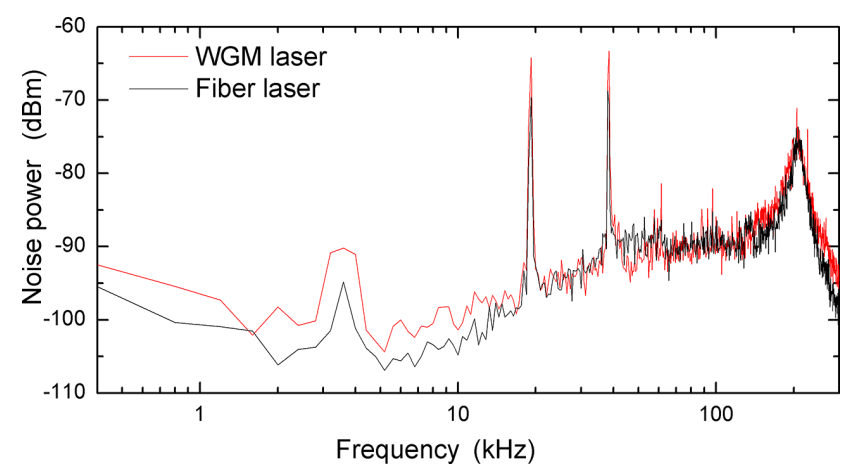

Figure 2. In-loop frequency spectrum of the error signal of the PDH locking for EDFL (black curve) and WGM laser (red curve).

\section{Measurement of SD NICE-OHMS signals}

Two acetylene transitions, one at $1531 \mathrm{~nm}$ and another at $1538 \mathrm{~nm}$, were addressed by the two lasers while sD signals were monitored. Figure 3 shows, by the black and red curves, two sD NICE-OHMS signals, from 13.3 Pa total pressure and $1000 \mathrm{ppm}$ concentration of $\mathrm{C}_{2} \mathrm{H}_{2}$, detected at the dispersion phase by the EDFL and WGM lasers, respectively. The pressure leaking rate for the cavity was smaller than $0.00133 \mathrm{~Pa}$ after it was closed by the vacuum valve (Leycon 215379, Oerlikon, Germany).

The EDFL addressed the $\mathrm{P}_{10}(\mathrm{e})$ transition of the $v_{1}+v_{3}$ band of $\mathrm{C}_{2} \mathrm{H}_{2}$ at $1530.976 \mathrm{~nm}$, which has a line strength of $4.0 \times 10^{-21} \mathrm{~mol} \mathrm{~cm}$ and a dipole moment of $8.24 \mathrm{mD}$. The saturation power under the pressure of $13.3 \mathrm{~Pa}$ was around

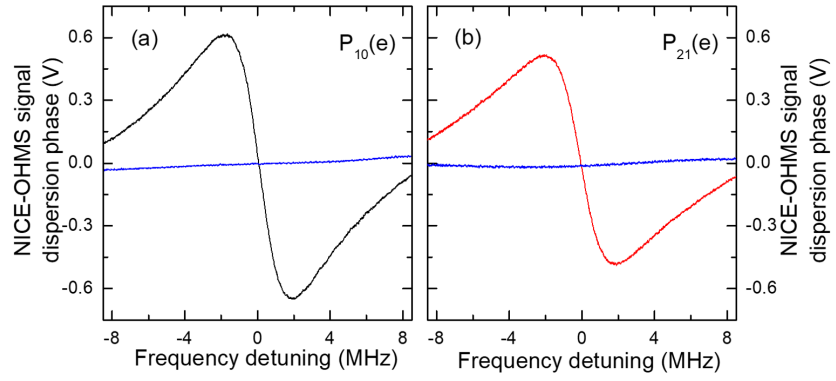

Figure 3. The sD NICE-OHMS signals detected at dispersion phase on the $\mathrm{P}_{10}(\mathrm{e})$ and $\mathrm{P}_{21}(\mathrm{e})$ transitions from $13.3 \mathrm{~Pa}$ of acetylene detection by an EDFL and a WGM laser, respectively. The blue lines in both panels are the background signals when the cavity was evacuated.

$700 \mathrm{~mW}$ (Foltynowicz et al., 2008b). The FWHM of the sD NICE-OHMS signal was about $3.5 \mathrm{MHz}$. It can be noted that by assuming that the transit time broadening is $188 \mathrm{kHz}$ and the degree of saturation is close to 6 (for an incident power of $5.86 \mathrm{~mW}$ ), it can be estimated that the pressure broadening should be around $1.05 \mathrm{MHz}$. This is inconsistent with the calculated broadening of $0.64 \mathrm{MHz}$ from the pressure broadening coefficient provided by the HITRAN database (a similar disagreement has previous been reported; Foltynowicz et al., 2008a). The frequency scan range, which is $16 \mathrm{MHz}$, is limited by the performance of VCO1 (whose resolution is proportional to the total scanning range). The degree of saturation was estimated from the ratio of amplitudes of $\mathrm{sD}$ and Db signals (Axner et al., 2008). 
The WGM laser addressed the $\mathrm{P}_{21}$ (e) transition of the $v_{1}+$ $\nu_{3}$ band of $\mathrm{C}_{2} \mathrm{H}_{2}$ at $1538.058 \mathrm{~nm}$, which has a line strength of $3.4 \times 10^{-21} \mathrm{~mol} \mathrm{~cm}$ and a dipole moment of $7.94 \mathrm{mD}$. The corresponding saturation power under the pressure of $13.3 \mathrm{~Pa}$ was around $800 \mathrm{~mW}$. The FWHM of the SD NICE-OHMS signal was about $3.8 \mathrm{MHz}$. By assuming both the similar transit time broadening and degree of saturation as above (for an incident power of $5.92 \mathrm{~mW}$ ), it can be estimated that the pressure broadening of this transition should be around $1.15 \mathrm{MHz}$.

The signal-to-noise ratios for the two sD signals, in Fig. 3, were similar, assessed to 380 , indicating detection sensitivities of $1.0 \times 10^{-9}$ and $0.9 \times 10^{-9} \mathrm{~cm}^{-1}$. The sensitivities are around 4 orders of magnitude worse than the best results in the field (Zhao et al., 2018), the reasons for which are the adopted lower finesse cavity, not fully optimized optical system and the different evaluation method of sensitivity (by evaluating the Allan variance of the fitted amplitude of long-term background measurements by a NICE-OHMS lineshape in all other EDFL-based NICE-OHMS papers). The blue lines in Fig. 3 show the background NICE-OHMS signals measured from an empty cavity that originates from etalons generated between optical components.

\section{Laser locking to the SD NICE-OHMS signal}

The frequency of each laser was then locked to the center of the addressed transition by feeding the sD signal back to the cavity PZT. The performance of this frequency stabilization was assessed with an Allan plot of the frequency deviation estimated from the error signals, i.e., the sD NICE-OHMS signals, calibrated by the slopes at the zero crossing point of the sD signals. The two uppermost panels in Fig. 4 show the frequency deviations for the EDFL and the WGM lasers measured over $4000 \mathrm{~s}$ with a sampling rate of $30 \mathrm{~Hz}$. It can be seen that both frequency deviations jitter around zero with little drift and that the deviations for the WGM laser are larger than those for the EDFL, consistent with the noise spectra shown in Fig. 2.

The lower panels in Fig. 4 show that for integration times up to $100 \mathrm{~s}$, the deviations in frequency have a white noise response, as is indicated by the dotted lines, which show a $\tau^{-1 / 2}$ dependence. The Allan plots furthermore show that, for an integration time of $1 \mathrm{~s}$, the relative frequency deviations of the EDFL (black dot line) and the WGM lasers (red dot line) are $9.4 \times 10^{-12}$ and $1.3 \times 10^{-11}$, respectively; however, for an integration time of $240 \mathrm{~s}$, the relative frequency deviations are $8.3 \times 10^{-13}$ and $7.5 \times 10^{-13}$, respectively (which corresponds to frequency stabilities of 0.16 and $0.15 \mathrm{kHz}$ ). The upturn of Allan deviation is due to the not fully suppressed residual amplitude modulation of fiber EOM, the imperfect design of PDH servo and etalon noise in the beam path.

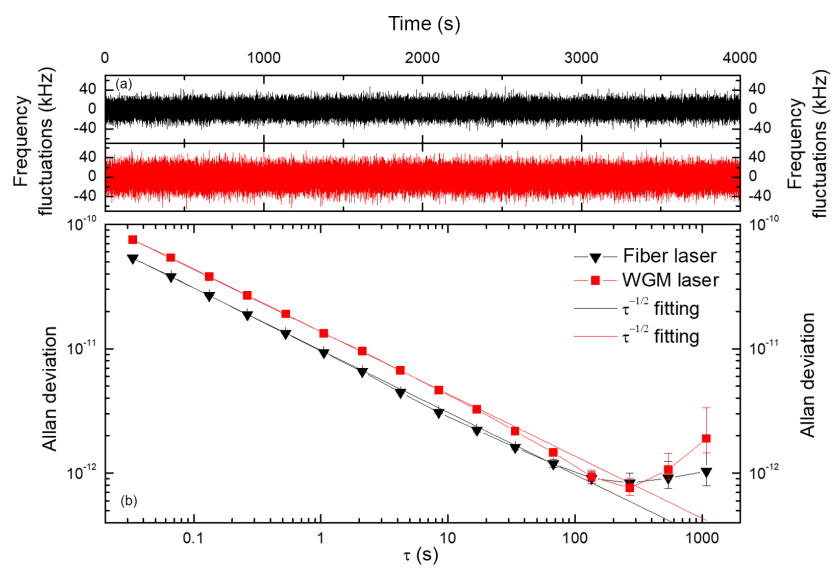

Figure 4. (a) The frequency deviation obtained from sD signals measured over $4000 \mathrm{~s}$ for the EDFL (black curve) and the WGM laser (red curve); (b) the Allan deviation of the frequency deviation for the EDFL (black curve) and the WGM laser (red curve). The dotted lines correspond to the white noise response.

However the laser frequency locking performance is strongly influenced by the amplitude and line width of the sD NICE-OHMS signal, which will be changed by the gas pressure and intracavity power. Although the locking results in the paper have satisfied the requirements of atmospheric lidar applications, if the NICE-OHMS system is optimized, an even better result can be expected.

\section{Conclusions}

We have demonstrated the construction of a universal NICEOHMS instrumentation for $\mathrm{SD}$ spectroscopy for the potential application in atmospheric lidar that is based on a single external frequency actuator, a fiber-coupled optical singlesideband electro-optic modulator (f-SSM), to control and detune the laser frequency. The universality of the instrumentation has been tested by alternatively incorporating it into an EDFL and a WGM laser. It has been demonstrated that the same locking system, with a given PID servo, could be used to lock both lasers to a given cavity, with the same bandwidth $(200 \mathrm{kHz})$. The use of a single external frequency actuator can eliminate the individual design of servos for each laser, which normally need to be specifically tailored for each laser. It also lowers the requirement of laser tunability for the spectrum measurement. It therefore significantly expands the applicability of the NICE-OHMS technique. Two $\mathrm{C}_{2} \mathrm{H}_{2}$ transitions were addressed by sD spectroscopy for which the laser frequency was tuned by alteration of the radio frequency to the f-SSM. Finally, the two laser frequencies were locked to the transition centers of the sD NICE-OHMS features, resulting in relative frequency stabilizations of $8.3 \times 10^{-13}$ and $7.5 \times 10^{-13}$ for an integration time of $240 \mathrm{~s}$. Although the frequency stability can satisfy the requirements of a real li- 
dar system, the whole setup should be miniaturized and a seismic design is necessary before it is applied to the real field. Conversely, if this setup is applied to the detection of $\mathrm{CO}_{2}, \mathrm{CH}_{4}$ or other gases, the saturation power of the target molecular transition, as a function of dipole moment, laser beam waist and total pressure, should be determined firstly to choose a suitable laser and a proper cavity design. The transition dipole moment is the function of its line strength, lifetime and partition function (Ma et al., 2008). Normally, for a weak absorption line with the saturation power on the order of hundreds of watts, a cavity with a finesse of more than 10000 and a laser with its line width of the order of kilohertz are required. The design of robust and universal sD NICE-OHMS instrumentation opens up a new application in atmospheric lidar.

Data availability. The data of Figs. 2, 3 and 4 can be found in the Supplement.

Supplement. The supplement related to this article is available online at: https://doi.org/10.5194/amt-12-1807-2019-supplement.

Author contributions. YZ and JL contributed the central idea, analyzed most of the data, and wrote the initial draft of the paper. SG analyzed the data, and GZ and WM developed the idea of the study. The remaining authors contributed to refining the ideas, carrying out additional analyses, and finalizing this paper.

Competing interests. The authors declare that they have no conflict of interest.

Special issue statement. This article is part of the special issue "Advances in cavity-based techniques for measurements of atmospheric aerosol and trace gases". It is not associated with a conference.

Acknowledgements. The work was supported by the National Key R\&D Program of China (grant no. 2017YFA0304203), Changjiang Scholars and Innovative Research Team in University of Ministry of Education of China (grant no. IRT_17R70), the Fund for Shanxi "1331 Project" Key Subjects Construction, 111 project (grant no. D18001), the National Natural Science Foundation of China (grant nos. 61675122, 61875107, 61875108, 11704236, 61475093 and 61775125), the Research Project Supported by Shanxi Scholarship Council of China (2017-016), the Program for the Outstanding Innovative Teams of Higher Learning Institutions of Shanxi and Open Research fund of Key Laboratory of Atmospheric Optics, Chinese Academy of Sciences (JJ-2018-02). Ove Axner would also like to acknowledge support from the Swedish Research Foundation (Vetenskapsrådet, grant no. 621-2015-04374).
Review statement. This paper was edited by Dean Venables and reviewed by three anonymous referees.

\section{References}

Axner, O., Ma, W., and Foltynowicz, A.: Sub-Doppler dispersion and noise-immune cavity-enhanced optical heterodyne molecular spectroscopy revised, J. Opt. Soc. Am. B, 25, 1166-1177, 2008.

Axner, O., Ehlers, P., Foltynowicz, A., Silander, I., and Wang, J.: NICE-OHMS - Frequency Modulation Cavity-Enhanced Spectroscopy - Principles and Performance, in: Cavity-Enhanced Spectroscopy and Sensing, edited by: Loock, H. P. and Gagliardi, G., Springer Series in Optical Sciences, Springer Verlag, Berlin, 2014.

Bell, C. L., Hancock, G., Peverall, R., Ritchie, G. A. D., van Helden, J. H., and van Leeuwen, N. J.: Characterization of an external cavity diode laser based ring cavity NICE-OHMS system, Opt. Express, 17, 9834-9839, 2009.

Chen, T. L. and Liu, Y. W.: Noise-immune cavity-enhanced optical heterodyne molecular spectrometry on $\mathrm{N}_{2} \mathrm{O} 1.283 \mu \mathrm{m}$ transition based on a quantum-dot external-cavity diode laser, Opt. Lett., 40, 4352-4355, 2015.

Chen, T. L. and Liu, Y. W.: Sub-Doppler resolution near-infrared spectroscopy at $1.28 \mu \mathrm{m}$ with the noise-immune cavity-enhanced optical heterodyne molecular spectroscopy method, Opt. Lett., 42, 2447-2450, 2017.

Delabachelerie, M., Nakagawa, K., and Ohtsu, M.: Ultranarrow ${ }^{13} \mathrm{C}_{2} \mathrm{H}_{2}$ saturated absorption lines at $1.5 \mu \mathrm{m}$, Opt. Lett., 19, 840 842, 1994.

DeVoe, R. G. and Brewer, R. G.: Laser frequency division and stabilization, Phys. Rev. A, 30, 2827-2829, 1984.

Dinesan, H., Fasci, E., Castrillo, A., and Gianfrani, L.: Absolute frequency stabilization of an extended-cavity diode laser by means of noise-immune cavity-enhanced optical heterodyne molecular spectroscopy, Opt. Lett., 39, 2198-2201, 2014.

Dinesan, H., Fasci, E., D’Addio, A., Castrillo, A., and Gianfrani, L.: Characterization of the frequency stability of an optical frequency standard at $1.39 \mu \mathrm{m}$ based upon noise-immune cavityenhanced optical, Opt. Express, 23, 1757-1766, 2015.

Drewer, R. W. P., Hall, J. L., Kowalski, F. V., Hough, J., Ford, F. M., Munley, A. J., and Ward, H.: Laser phase and frequency stabilization using an optical resonator, Appl. Phys. B, 31, 97-105, 1983.

Ehlers, P.: Further development of NICE-OHMS - an ultra-sensitive frequency-modulated cavity-enhanced laser-based spectroscopic technique for detection of molecules in gas phase, 2014, $\mathrm{PhD}$ Thesis, Department of Physics, Umeå university, Umeå, Sweden, 2014.

Ehlers, P., Silander, I., Wang, J., and Axner, O.: Fiber-laser-based noise-immune cavity-enhanced optical heterodyne molecular spectrometry instrumentation for Doppler-broadened detection in the $10^{-12} \mathrm{~cm}^{-1} \mathrm{~Hz}^{-1 / 2}$ region, J. Opt. Soc. Am. B, 29, 13051315, 2012.

Ehlers, P., Johansson, A. C., Silander, I., Foltynowicz, A., and Axner, O.: Use of etalon-immune distances to reduce the influence of background signals in frequency-modulation spectroscopy and noise-immune cavity-enhanced optical heterodyne 
molecular spectroscopy, J. Opt. Soc. Am. B, 31, 2938-2945, 2014.

Ehret, G., Kiemle, C., Wirth, M., Amediek, A., Fix, A., and Houweling, S.: Space-borne remote sensing of $\mathrm{CO}_{2}, \mathrm{CH}_{4}$, and $\mathrm{N}_{2} \mathrm{O}$ by integrated path differential absorption lidar: a sensitivity analysis, Appl. Phys. B., 90, 593-608, 2008.

Foltynowicz, A.: Fiber-laser-based noise-immune cavity-enhanced optical heterodyne molecular spectrometry, 2009, PhD Thesis, Department of Physics, Umeå University, Umeå, Sweden, 2009.

Foltynowicz, A., Ma, W., and Axner, O.: Characterization of fiberlaser-based sub-Doppler NICE-OHMS for quantitative trace gas detection, Opt. Express, 16, 14689-14702, 2008a.

Foltynowicz, A., Ma, W. G., Schmidt, F. M., and Axner, O.: Doppler-broadened noise-immune cavity-enhanced optical heterodyne molecular spectrometry signals from optically saturated transitions under low pressure conditions, J. Opt. Soc. Am. B, 25, 1156-1165, 2008b.

Foltynowicz, A., Wang, J., Ehlers, P., and Axner, O.: Distributedfeedback-laser-based NICE-OHMS in the pressure-broadened regime, Opt. Express, 18, 18580-18591, 2010.

Fox, R. W., Oates, C. W., and Hollberg, L. W.: Stabilizing diode lasers to high-finesse cavities, in: Cavity-Enhanced Spectroscopies, edited by: van Zee, R. D. and Looney, J. P., Experimental Methods in the Physical Sciences, Elsevier Science, New York, 2002.

Gatti, D., Gotti, R., Sala, T., Coluccelli, N., Belmonte, M., Prevedelli, M., Laporta, P., and Marangoni, M.: Wide-bandwidth Pound-Drever-Hall locking through a single-sideband modulator, Opt. Lett., 40, 5176-5179, 2015.

Hausmaninger, T., Silander, I., and Axner, O.: Narrowing of the linewidth of an optical parametric oscillator by an acousto-optic modulator for the realization of mid-IR noise-immune cavityenhanced optical heterodyne molecular spectrometry down to $10^{-10} \mathrm{~cm}^{-1} \mathrm{~Hz}^{-1 / 2}$, Opt. Express, 23, 33641-33655, 2015.

Ishibashi, C. and Sasada, H.: Highly sensitive cavity-enhanced subDoppler spectroscopy of a molecular overtone band with a 1.66 $\mu \mathrm{m}$ tunable diode laser, Jpn. J. Appl. Phys. 1, 38, 920-922, 1999.

Ishibashi, C. and Sasada, H.: Near-infrared laser spectrometer with sub-Doppler resolution, high sensitivity, and wide tunability: A case study in the $1.65-\mu \mathrm{m}$ region of $\mathrm{CH}_{3} \mathrm{I}$ spectrum, J. Mol. Spectrosc., 200, 147-149, 2000.

Loayssa, A., Benito, D., and Garde, M. J.: Single-sideband suppressed-carrier modulation using a single-electrode electrooptic modulator, IEEE Photonic. Tech. L., 13, 869-871, 2001.

Ma, L. S., Ye, J., Dube, P., and Hall, J. L.: Ultrasensitive frequencymodulation spectroscopy enhanced by a high-finesse optical cavity: theory and application to overtone transitions of $\mathrm{C}_{2} \mathrm{H}_{2}$ and $\mathrm{C}_{2}$ HD, J. Opt. Soc. Am. B, 16, 2255-2268, 1999.

Ma, W. G., Foltynowicz, A., and Axner, O.: Theoretical description of Doppler-broadened noise-immune cavity-enhanced optical heterodyne molecular spectroscopy under optically saturated conditions, J. Opt. Soc. Am. B, 25, 1144-1155, 2008.

Ma, W. G., Silander, I., Hausmaninger, T., and Axner, O.: Dopplerbroadened NICE-OHMS beyond the cavity-limited weak absorption condition - I. Theoretical description, J. Quant. Spectrosc. Ra., 168, 217-244, 2016.

Meras, P. J., Poberezhskiy, I. Y., Chang, D. H., Levin, J., and Spiers, G. D.: Laser frequency stabilization for coherent lidar applications using novel all-fiber gas reference cell fabrication tech- nique, 24th International Laser Radar Conference, Boulder, Colorado, 2008.

Moretti, L., Castrillo, A., Fasci, E., De Vizia, M. D., Casa, G., Galzerano, G., Merlone, A., Laporta, P., and Gianfrani, L.: Determination of the Boltzmann Constant by Means of Precision Measurements of $\mathrm{H}_{2}^{18} \mathrm{O}$ Line Shapes at $1.39 \mu \mathrm{m}$, Phys. Rev. Lett., 111, 060803, 2013.

Numata, K., Chen, J. R., Wu, S. T., Abshire, J. B., and Krainak, M. A.: Frequency stabilization of distributed-feedback laser diodes at $1572 \mathrm{~nm}$ for lidar measurements of atmospheric carbon dioxide, Appl. Optics, 50, 1047-1056, 2011.

Parthey, C. G., Matveev, A., Alnis, J., Bernhardt, B., Beyer, A., Holzwarth, R., Maistrou, A., Pohl, R., Predehl, K., Udem, T., Wilken, T., Kolachevsky, N., Abgrall, M., Rovera, D., Salomon, C., Laurent, P., and Hansch, T. W.: Improved Measurement of the Hydrogen 1S-2S Transition Frequency, Phys. Rev. Lett., 107, 203001, 2011.

Saraf, S., Berceau, P., Stochino, A., Byer, R., and Lipa, J.: Molecular frequency reference at $1.56 \mu \mathrm{m}$ using a ${ }^{12} \mathrm{C}^{16} \mathrm{O}$ overtone transition with the noise-immune cavity-enhanced optical heterodyne molecular spectroscopy method, Opt. Lett., 41, 2189-2192, 2016.

Schmidt, F. M., Foltynowicz, A., Ma, W., Lock, T., and Axner, O.: Doppler-broadened fiber-laser-based NICE-OHMS - Improved detectability, Opt. Express, 15, 10822-10831, 2007.

Schmidt, F. M., Ma, W., Foltynowicz, A., and Axner, O.: Highly sensitive dispersion spectroscopy by probing the free spectral range of an optical cavity using dual-frequency modulation, Appl. Phys. B., 101, 497-509, 2010.

Silander, I., Ehlers, P., Wang, J., and Axner, O.: Frequency modulation background signals from fiber-based electro optic modulators are caused by crosstalk, J. Opt. Soc. Am. B, 29, 916-923, 2012.

Talicska, C. N., Porambo, M. W., Perry, A. J., and McCall, B. J.: Mid-infrared concentration-modulated noise-immune cavityenhanced optical heterodyne molecular spectroscopy of a continuous supersonic expansion discharge source, Rev. Sci. Instrum., 87, 063111, 2016.

Tarallo, M. G., Poli, N., Schioppo, M., Sutyrin, D., and Tino, G. M.: A high-stability semiconductor laser system for a ${ }^{88}$ Sr-based optical lattice clock, Appl. Phys. B, 103, 17-25, 2011.

Taubman, M. S., Myers, T. L., Cannon, B. D., and Williams, R. M.: Stabilization, injection and control of quantum cascade lasers, and their application to chemical sensing in the infrared, Spectrochim. Acta A, 60, 3457-3468, 2004.

van Leeuwen, N. J. and Wilson, A. C.: Measurement of pressurebroadened, ultraweak transitions with noise-immune cavityenhanced optical heterodyne molecular spectroscopy, J. Opt. Soc. Am. B, 21, 1713-1721, 2004.

Ye, J., Ma, L. S., and Hall, J. L.: Sub-Doppler optical frequency reference at $1.064 \mu \mathrm{m}$ by means of ultrasensitive cavity-enhanced frequency modulation spectroscopy of a $\mathrm{C}_{2} \mathrm{HD}$ overtone transition, Opt. Lett., 21, 1000-1002, 1996.

Zhao, G., Hausmaninger, T., Ma, W., and Axner, O.: Whisperinggallery-mode laser-based noise-immune cavity-enhanced optical heterodyne molecular spectrometry, Opt. Lett., 42, 3109-3112, 2017. 
Zhao, G., Hausmaninger, T., Ma, W., and Axner, O.: Shot-noiselimited Doppler-broadened noise-immune cavity-enhanced optical heterodyne molecular spectrometry, Opt. Lett., 43, 715-718, 2018. 